\title{
Fabrication and Ferroelectric Properties of $\mathrm{BiFeO}_{3} / \mathrm{BaTiO}_{3}$ Heterostructures
}

\author{
M. Aleszkiewicz, K. Dybko, E. Dynowska, P. Dluzewski and P. Przyslupski* \\ Institute of Physics, Polish Academy of Sciences, al. Lotników 32/46, 02-668 Warsaw, Poland
}

\begin{abstract}
We report on preparation and electrical characterization of the epitaxial $\mathrm{BaTiO}_{3}(\mathrm{BTO}), \mathrm{BiFeO}_{3}(\mathrm{BFO})$ thin films and BFO/BTO bi- and multilayers, grown on (001) $\mathrm{SrTiO}_{3}(\mathrm{STO})$ and $\left(\mathrm{LaAlO}_{3}\right)_{0.3}\left(\mathrm{Sr}_{2} \mathrm{TaAlO}_{6}\right)_{0.7}(\mathrm{LSAT})$ substrates. The ferroelectric properties were characterized using the electric force microscopy method to image and switch the electric domains. This fabrication process opens the routes towards wide study of magnetoelectric effect in complex oxide heterostructures.
\end{abstract}

DOI: 10.12693/APhysPolA.130.511

PACS/topics: 75.85.+t, 77.80.-e, 77.84.-s, 77.84.Bw

\section{Introduction}

There has been a long-standing research interest in the integration of functional oxides in artificial heterostructures not only for fundamental research but also for potential applications. The magnetoelectric (ME) properties of multiferroic (MF) materials demonstrate a coupling between ferroelectric (FE) and ferromagnetic (FM) order parameters which provides the possibility to control FE properties by magnetic field and vice versa. Several mechanisms are responsible for intrinsic ME effect $[1,2]$. ME coupling can also occur at FM/FE interfaces where its mechanism is mediated by strain (magnetostriction and piezoelectricity), charge (sensitivity of magnetic state on charge accumulation) or exchange bias (coupling of antiferromagnetic domain walls to ferroelectric ones) [3]. ME effect was also observed in multiferroic tunnel junctions composed of two FM electrodes separated by thin FE barrier [4]. At the atomic level it is the interface bonding which is the source of ME effect in heterostructures.

Bismuth ferrite (BFO) has been studied extensively as one of a few single-phase room-temperature multiferroics (ferroelectric and antiferromagnetic with $1103 \mathrm{~K}$ FE Curie and $643 \mathrm{~K}$ Néel temperature, respectively) with high ferroelectric polarization [5]. Barium titanate (BTO) is one of the important ferroelectric perovskites in the tetragonal form [6] with FE Curie temperature $T_{\mathrm{C}} \approx 390 \mathrm{~K}$.

The multilayered-type heterostructures offer more degrees of freedom to tune ME properties [2]. Such composite ferromagnetic/multiferroic/ferroelectric heterostructures provide opportunities for studying ME effect at nanoscale as well as for application in magnetoelectric devices such as ferroelectric tunnel junctions.

In this paper we report the studies on the preparation of BTO and BFO thin films deposited on STO and LSAT

\footnotetext{
*corresponding author; e-mail: przys@ifpan.edu.pl
}

substrates as well as $\mathrm{BFO} / \mathrm{BTO}$ bilayers and $\mathrm{BFO} / \mathrm{BTO}$ multilayers. The combined $\mathrm{BFO} / \mathrm{BTO}$ structures might serve as platform for studying the ME effect, where ferroelectric properties of the electrode could be tuned by varying the thicknesses of $\mathrm{BFO}$ and $\mathrm{BTO}$ systems in multilayered structure.

\section{Experimental}

The BTO, BFO thin films, BFO/BTO bilayers and $\mathrm{BFO} / \mathrm{BTO}$ multilayers were deposited onto (001) STO and (001) LSAT substrates by RF high pressure sputtering in 1.5 mbar oxygen pressure at $680-750^{\circ} \mathrm{C}$ substrate temperature $[7,8]$.

X-ray diffraction measurements have been performed with the use of X-ray diffractometer (Philips X'Pert MPD Pro Alpha1, $\mathrm{Cu} K_{\alpha_{1}}$ radiation of $\lambda=1.540598 \AA$ ).

The morphology of the surface and electric properties of heterostructures were investigated by the scanning probe microscopy using a Nanoscope MultiMode system at ambient conditions. As conductive probes the $\mathrm{PtCo}-$ coated Bruker cantilevers were used. Atomic force microscopy (AFM) surface images were taken in dynamic tapping mode. Roughness parameters given in the further text to characterize the sample surface were calculated for $2 \times 2 \mu \mathrm{m}^{2}$ relatively smooth area which was chosen from $10 \times 10 \mu \mathrm{m}^{2}$ AFM image. Following standard parameters are to be compared: the maximum height difference between the highest and the lowest point on the surface $z_{\max } ; r m s$ surface roughness $R_{\mathrm{rms}}$ (standard deviation of the values within the chosen area); mean roughness $R_{a}$ (arithmetic average from the deviations from the center plane). Local electric surface potential was sensed via long-ranging electrostatic forces resulting from a potential offset between the tip and the surface by electric force microscopy (EFM). The two-pass technique [9] with phase locked loop (PLL) was used and the cantilever frequency shift was detected. According to the formula (which is valid for small tip amplitudes in direction $z$ perpendicular to the surface): $\Delta \omega \sim \partial F_{e l} / \partial z=$ $-\frac{1}{2}\left(\partial^{2} C / \partial z^{2}\right) U^{2}$, the frequency shift $\Delta \omega$ is proportional 
to the electrical force gradient $\partial F_{e l} / \partial z$ and does not depend on mechanical properties of the tip. Due to the lack of knowledge of the tip-sample capacitance $C$ (which depends on the shape and complex dynamics of the tip) the method yields only qualitative results on surface potential $U$. Moreover, the field distribution between the tip and the thin film (or multilayered) counterelectrode is quite complex and quantitative data may not be extracted.

EFM was also used for charge injection into the surface. The procedure of localized charge deposits consists of bringing the conductive tip to the closer proximity of the sample surface (by diminishing the tip amplitude) and simultaneous application of a relatively high bias voltage (in the range from $-10 \mathrm{~V}$ to $+10 \mathrm{~V}$ ) between the tip and sample. The time duration and amplitude of the voltage pulse can be tuned and the typical result of such charge writing is a single-volt difference of surface potential with respect to untreated areas. The electric field can be as high as $10^{6} \mathrm{~V} / \mathrm{mm}$ when a smallradius tip of is placed within about $1 \mathrm{~nm}$ to the surface. Due to the high inhomogeneity of the electric field induced by the tip, the field intensity drops rapidly with the surface thickness. Therefore, only the outer surface of several $\mu \mathrm{m}$ thickness is polarized in this procedure which is sufficient for the layers measured in this work. The tip is guided along a defined path which creates regions of certain negative/positive polarization. In this work, rectangular-shaped regions of given width, periodicity and/or aspect ratio are created. The subsequent EFM measurements detect the injected carriers and their volatility i.e. possible diffusion or natural discharge due to charge transfer between the tip and the sample.

The cross-section images of heterostructures were acquired with transmission electron microscope (TEM) Helios-NanoLab 600. The system is equipped with energy-dispersive X-ray spectrometer (EDX) which was used for elemental analysis of the individual layers.

\section{Results}

An example of induced charge pattern is shown in EFM image in Fig. 1 for the ferroelectric BTO film on LSAT substrate. The opposite poling of neighboring regions was obtained by alternate sign changes of applied voltage $\pm 10 \mathrm{~V}$. The long-term stability (several days) of the EFM signal confirms the induction of permanent dipole moments in BTO.

The multiferroic BFO/BTO bilayers (BFO deposited first) were grown on LSAT (Fig. 2) and STO (Fig. 4). AFM characterization shows the good crystallinity of surfaces for both these cases. However, bilayers grown on STO show clear texture of the surface, with parallel topographical structures of approximately $200 \mathrm{~nm}$ width. The longitudinal grooves are visible in topography image in Fig. 4a when imaged at 35 deg scan angle. This topographical orientation influences (increases) the roughness parameters which is thus distinctly smaller for

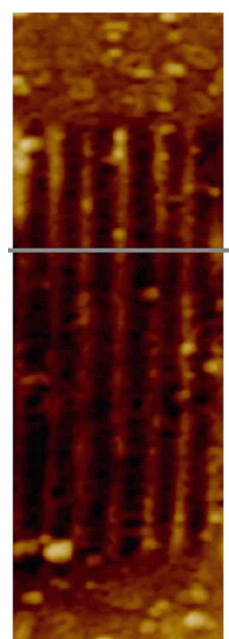

a)

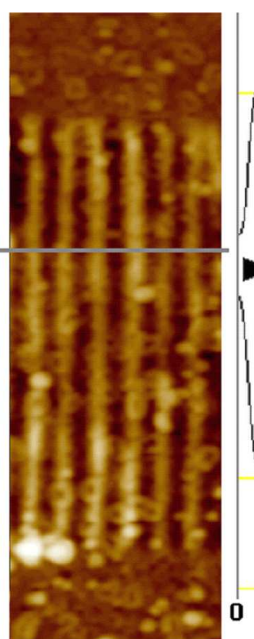

b)

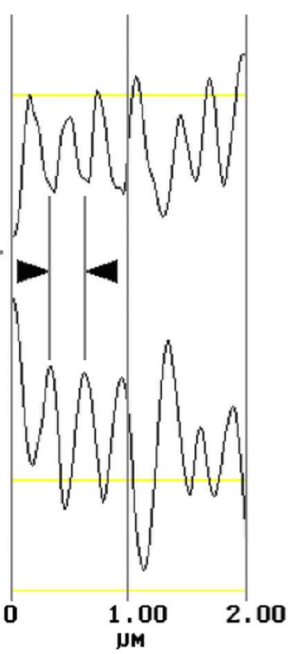

c)
Fig. 1. BTO on LSAT substrate: EFM of narrow stripes poled by alternately changing voltage. The surface area is $2 \times 6 \mu \mathrm{m}$ and the periodicity of the written pattern is $300 \mathrm{~nm}$; a) and b) are measured with opposite tip polarization; c) are cross-section lines of a) and b), the marked distance is $300 \mathrm{~nm}$.

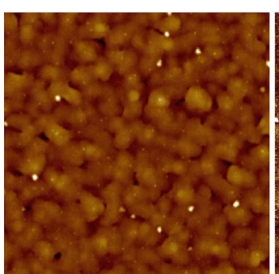

a)

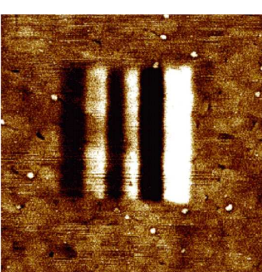

b)

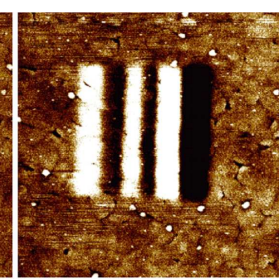

c)
Fig. 2. BFO/BTO bilayer on LSAT substrate, surface area $4.2 \times 4.2 \mu \mathrm{m}$; a) AFM image, z scale $30 \mathrm{~nm}$; b) and c) EFM of $2 \times 2 \mu \mathrm{m}$ square poled in pattern $400 / 350 / 250 / 250 / 350 / 400 \mathrm{~nm}$ measured with opposite tip polarizations and lift height $20 \mathrm{~nm}$.

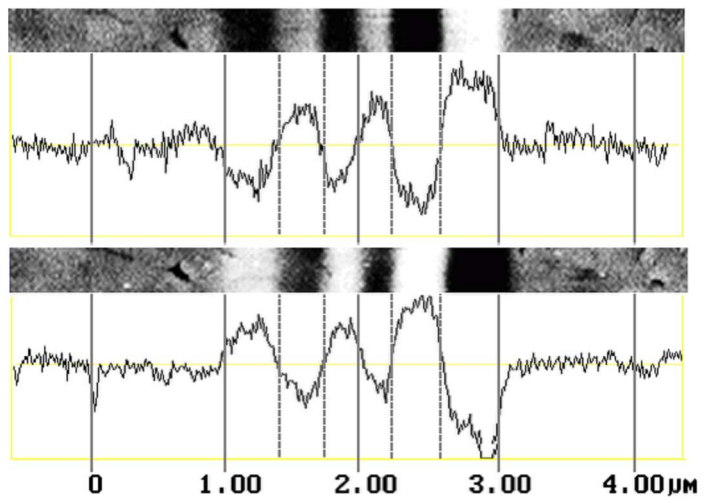

Fig. 3. BFO/BTO bilayer on LSAT substrate: corresponding EFM maps measured with opposite tip polarization and their section lines. The written pattern consisting of the $400 / 350 / 250 / 250 / 350 / 400 \mathrm{~nm}$ stripes has the total width $2 \mu \mathrm{m}$. The polarization direction reversal occurs in $30-50 \mathrm{~nm}$ wide transition regions. 
the structure grown on LSAT than on STO. The roughness parameters are as follows: $z_{\max }=16 \mathrm{~nm} / 81 \mathrm{~nm}$; $R_{\mathrm{rms}}=1.5 \mathrm{~nm} / 8.9 \mathrm{~nm}$; mean $R_{a}=1.2 \mathrm{~nm} / 6.6 \mathrm{~nm}$ for LSAT/ STO substrates, respectively.

Bulk BFO is known to have a rhombohedrally distorted perovskite structure assigned to the space group R3c with hexagonal lattice parameters $a_{\text {hex }}=5.579 \AA$, $c_{\text {hex }}=13.869 \AA[10]$ or the other rhombohedral phase of R-3m type with hexagonal parameters $a_{\text {hex }}=5.580 \AA$, $c_{\text {hex }}=6.930 \AA$ [11]. Note that the $c$-parameter in this phase is equal to half of the $c$-parameter in the first phase. Bulk BTO has the tetragonal structure of $P 4 \mathrm{~mm}$ type with $a=3.994 \AA$ and $c=4.038 \AA$ lattice parameters [12]. The analysis of X-ray diffraction patterns shows that the BFO layer grows in [101] direction in hexagonal unit cell both for LSAT (Fig. 5) and STO (Fig.6) substrates (the hexagonal [101] direction is equivalent to [001] direction in pseudo-cubic unit cell). The BTO layer grows on BFO layer in [001] tetragonal direction for both substrates. However, the BFO and BTO lattice spacings are different for the two substrates. In the BFO layer grown on LSAT (Fig. 5) the measured lattice spacings in [101] direction are $d_{101}=3.967 \AA, d_{202}=1.984 \AA$, $d_{303}=1.322 \AA$, what indicates that $\mathrm{BFO}$ layer is relaxed. The following BTO layer, with $c=4.080 \AA$, is compressively strained. In the case of BFO layer grown on STO (Fig. 6) the lattice spacings in [101] direction are $d_{101}=4.020 \AA, d_{202}=2.012 \AA, d_{303}=1.343 \AA$ what indicates that the $\mathrm{BFO}$ layer is not relaxed but compressively strained. The top BTO layer with $c=4.141 \AA$ is under compressive strain.

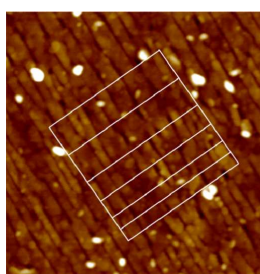

a)

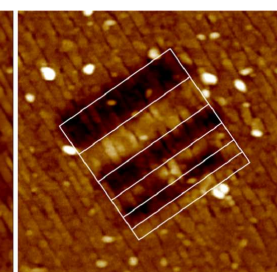

b)

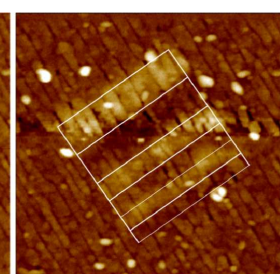

c)
Fig. 4. BFO/BTO bilayer on STO substrate, surface area $4 \times 4 \mu \mathrm{m}$; a) AFM image with z scale $100 \mathrm{~nm}$, showing lateral topographical structures of few hundreds $\mathrm{nm}$ width; b) and c) EFM of $2 \times 2 \mu \mathrm{m}$ square poled in pattern $2 \times 200,2 \times 300,2 \times 500 \mathrm{~nm}$ (from bottom to top) measured with opposite tip polarizations. The lines are drawn as a guide for the eye.

The differences in lattice mismatch between BFO layer-LSAT substrate $(2 \%)$ and BFO layer-STO substrate $(1 \%)$ which lead to relaxed and strained growth, respectively, are the reason for the differences in surface roughness values revealed by AFM: low for relaxed BFO layer on LSAT (Fig. 2) and large for strained BFO on STO substrate (Fig. 4).

BFO/BTO bilayers show no spontaneous preferential polarization direction on the surface but the poling results in a clear ferroelectric orientation. Possibly due to its smaller surface roughness, the induced charge pat-

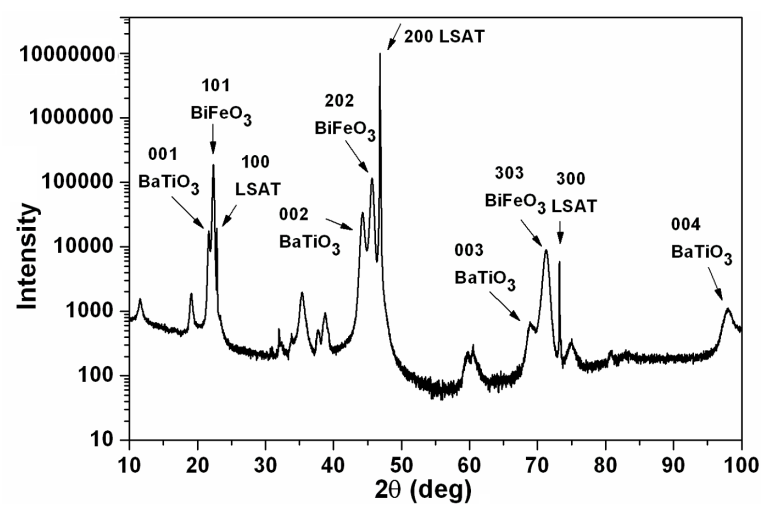

Fig. 5. X-ray diffraction pattern of BFO/BTO bilayer on LSAT substrate.

tern is more distinct and sharp on LSAT-grown sample. The width of a transition region between induced electrical domains is as narrow as $30 \mathrm{~nm}$ (Fig. 3) which is comparable to EFM tip resolution. The patterns written on STO-grown bilayers are more blurred, independent whether written in direction perpendicular (as in Fig. 4) or parallel (not shown) to topographical structures. In that last case the electrical domain is augmented to the topographical structure and therefore its width is about $200 \mathrm{~nm}$. The temporal stability of the injected carriers is comparable for both substrates and - independently of scan-induced discharging in consecutive scans - the surface potential reduces to immeasurable values within 1 day.

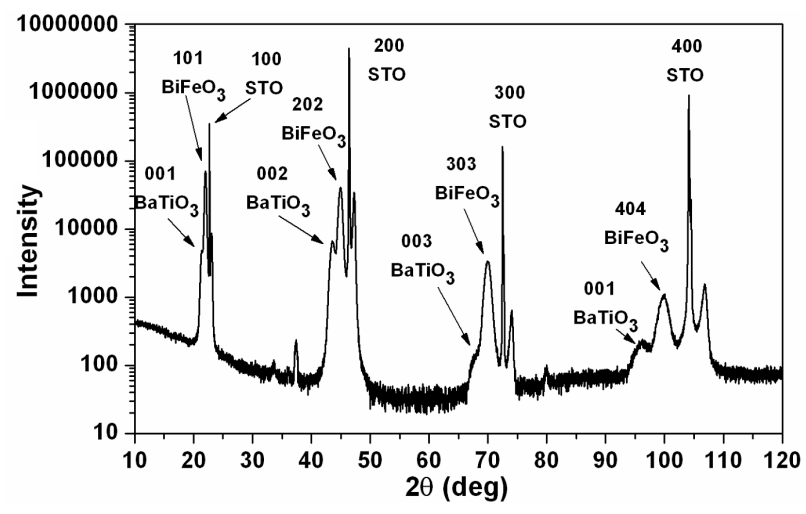

Fig. 6. X-ray diffraction pattern of BFO/BTO bilayer on STO substrate.

In attempt to enhance the magnetoelectric coupling more complex heterostructures: $\left[\begin{array}{lll}\text { BTO } & (10 \mathrm{~nm}) / \\ \text { BFO } & (10 & \mathrm{nm})\end{array}\right] \times 10$ (further referred to as small period $\mathrm{SP} \mathrm{ML}$ ) and [BTO $(30 \mathrm{~nm}) / \operatorname{BFO}(30 \mathrm{~nm})] \times 6$ multilayers (large period LP ML, respectively) were deposited. X-ray diffraction analysis shows that the ML heterostructures are not epitaxial, in contrary to that what was observed for single BTO and BFO thin films and BFO/BTO bilayers. For SP ML, AFM reveals perpendicularly oriented rectangular grains with very high values of roughness parameters (Fig. 7a). Typically they 


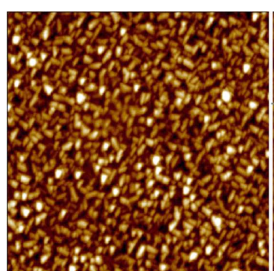

a)

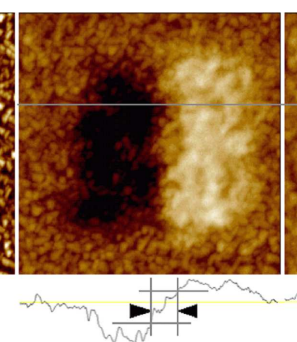

b)

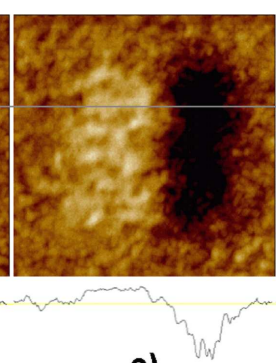

c)
Fig. 7. SP ML (i.e. [BTO $(10 \mathrm{~nm}) / \mathrm{BFO}(10 \mathrm{~nm})] \times 10)$ on STO substrate, surface area $10 \times 10 \mu \mathrm{m}$; a) AFM image, $z$ scale $100 \mathrm{~nm}$; b) poled square of area $5 \times 5 \mu \mathrm{m}$ (left part $+10 \mathrm{~V}$, right part $-10 \mathrm{~V}$ ) imaged with $-1 \mathrm{~V}$; c) imaged with $+1 \mathrm{~V}$; lift height $150 \mathrm{~nm}$. High charge diffusion occurs and there is no sharp boundary between + and - areas; the distance between markers in the section line is $1.0 \mu \mathrm{m}$.

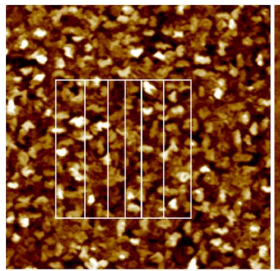

a)

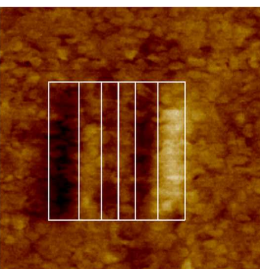

b)

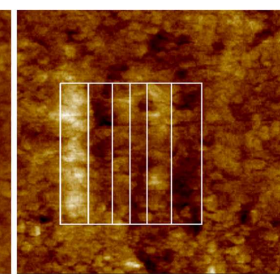

c)
Fig. 8. LP ML (i.e. [BTO $(30 \mathrm{~nm}) / \operatorname{BFO}(30 \mathrm{~nm}] \times 6)$ grown on LSAT substrate, surface area $4 \times 4 \mu \mathrm{m}$; a) AFM image, $z$ scale $25 \mathrm{~nm}$; b) and c) EFM of $2 \times 2 \mu \mathrm{m}$ square poled in pattern $400 / 350 / 250 / 250 / 350 / 400 \mathrm{~nm}$ measured with opposite tip polarizations and lift height $20 \mathrm{~nm}$. The lines are drawn as a guide for the eye.
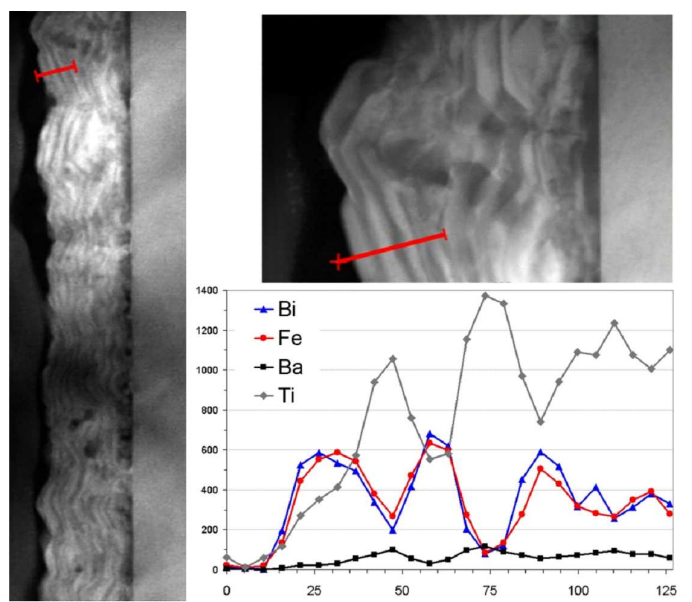

Fig. 9. HRTEM cross section topography and EDX composition of $\mathrm{BFO} / \mathrm{BTO}$ multilayer deposited on LSAT substrate. The length of the cross section line (bar) is $125 \mathrm{~nm}$. are: $z_{\max }=140 \mathrm{~nm} / 99 \mathrm{~nm} ; R_{\mathrm{rms}}=27 \mathrm{~nm} / 17 \mathrm{~nm}$; mean $R_{a}=22 \mathrm{~nm} / 14 \mathrm{~nm}$ for $\mathrm{SP} \mathrm{ML}$ grown on LSAT/STO, respectively. Apparently, the value of $z_{\max }$ is close to the nominal thickness of the whole stack of layers i.e. the total thickness of the MLs - particularly in the case of LSAT substrate. This suggests the island or polycrystalline growth of the successive layers and the sample may behave rather as a composite than a combined ferroelectric. It was shown [13] that composite films are rougher than layers due to the granular structure of BTO and BFO phases with nanoparticle grain size in the order of 40-100 nm (BTO) and 100-200 nm (BFO).

Because of the high surface roughness the charge injection by EFM is not site-precise. Additionally, the written paths have to be broad enough to enable their further detection with relatively large tip elevation in lift mode, adapted to the height of surface structures. The transition region between the stripes (i.e. the distance between FWHM values) of opposite polarity is approx. $1 \mu \mathrm{m}$ wide (Fig. 7 b,c). The time stability of poling is limited to one hour and after this time no EFM signal is measurable. The EFM signal volatility can be observed in two consecutive scans in Fig. 7b, c where the contrast decrease is about $40 \%$ in time interval of about $20 \mathrm{~min}$.

Despite the larger total thickness of LP ML, their roughness parameters of $\mathrm{z}_{\max }=35 \mathrm{~nm}, \mathrm{R}_{\mathrm{rms}}=5 \mathrm{~nm}$, mean $R_{a}=4 \mathrm{~nm}$ (LSAT substrate, Fig. 8a) are remarkably smaller than those for SP ML. Nevertheless, the charge injection efficiency is limited as in the case of SP ML. Because of smaller surface roughness, the pattern of $250 \mathrm{~nm}$ linewidth is still recognizable (Fig. 8b, c) but the time stability of the written paths is comparable to that of SP ML. This leads to the conclusion that in ML heterostructures randomly oriented small grains are formed. The polarization vectors of the grains cancel each other and this leads to low EFM signal when compared to that of bilayers.

The ferroelectric properties of the series of BTO-BFO heterostructures presented here allow for their use in devices with magnetoelectric effect. As mentioned above this could be realized at ferromagnetic - ferroelectric/ multiferroic surface or in tunnel junction with BTO-BFO barrier deposited on the BTO-BFO platform, where such a structure could increase the ME effect. According to our study, the increased number of $\mathrm{BFO} / \mathrm{BTO}$ repetitions impairs the integrity of individual layers. The expected enhancement of the ME effect due to duplication of interfaces is on the cost of their quality. Therefore better results of charge injection were achieved for bilayers than for ML structures. The individual layers in $\mathrm{BFO} / \mathrm{BTO}$ multilayer are strongly undulated due to compressive strain as shown in TEM cross-section image (Fig. 9). However, the EDX intensity spectrum confirms the sharpness of boundaries between individual layers. Abrupt changes in elemental composition indicate the good quality of interfaces. 


\section{Conclusions}

In summary, the ferroelectric properties of ferroelectric-multiferroic system could be tuned by varying thicknesses of heterostructures were deposited on various substrates: (001) STO and LSAT. Epitaxial growth was achieved for single- and bilayers, but $\mathrm{BFO} / \mathrm{BTO}$ multilayers showed granular structure with roughness comparable to layer thickness. The applicability of BFO/BTO heterostructures as ferroelectric platform for study the ME effect depends on their quality (i.e. electric polarizability) which is determined by the interface effects. Heterostructures demonstrate a good crystallinity, as manifested in distinct orientation of topographical structures. Low roughness is crucial for magnitude and stability of induced polarization, the best results were obtained for $\mathrm{BFO} / \mathrm{BTO}$ bilayers, where the non-volatile polarization was kept at least several days after poling. Bilayers grown on LSAT substrate with roughness values of the order of few $\mathrm{nm}$ seem to be the most appropriate as a FE platform. Multilayers show higher roughness than bilayers, what results in weaker and diffused polarization.

\section{References}

[1] K.F. Wang, J.-M. Liu, Z.F. Ren, Adv. Phys. 58, 321 (2009).

[2] C.A.F. Vaz, J. Hoffman, C.H. Ahn, R. Ramesh, Adv. Mater. 22, 2900 (2010).
[3] A. Bhattacharya, S.J. May, Ann. Rev. Mater. Res. 44, 65 (2014).

[4] Y.W. Yin, M. Raju, W.J. Hu, X.J. Weng, X.G. Li, Q. Li, J. Appl. Phys. 109, 07D915 (2011).

[5] J.T. Heron, D.G. Schlom, R. Ramesh, Appl. Phys. Rev. 1, 021303 (2014).

[6] R.E. Cohen, Nature 358, 136 (1992).

[7] K. Werner-Malento, A. Tsarou, P. Dluzewski, W. Paszkowicz, R. Minikayev, M. Sawicki, K. van der Beek, M. Konczykowski, P. Przyslupski, Acta Phys. Pol. A 115, 95 (2009).

[8] P. Przyslupski, A. Tsarou, P. Dłużewski, W. Paszkowicz, R. Minikayev, K. Dybko, M. Sawicki, B. Dabrowski, C. Kimball, Supercond. Sci. Technol. 19, S38 (2006).

[9] H. Bluhm, A. Wadas, R. Wiesendanger, A. Roshko, J.A. Aust, D. Nam, Appl. Phys. Lett. 71, 146 (1997).

[10] F. Kubel, H. Schmidt, Acta Crystallogr. B 46, 698 (1990).

[11] JCPDS, 14-0181.

[12] JCPDS, 05-0626.

[13] M. Lorenz, V. Lazenka, P. Schwinkendorf, F. Bern, M. Ziese, H. Modarresi, A. Volodin, M.J. Van Bael, K. Temst, A. Vantomme, M. Grundmann, J. Phys. D Appl. Phys. 47, 135303 (2014). 